Mathematics and Mechanics of Solids, 11(6), 563-574

\title{
Existence, uniqueness and regularity of solutions for a thermomechanical model of shape memory alloys
}

\author{
Tudor Ratiu ${ }^{1} \quad$ Aida Timofte Tlad Timofte $^{3}$
}

\begin{abstract}
This paper considers a thermomechanical model describing the behavior of shape memory alloys (SMA). This new model (see [3]) takes into account the nonisothermal character of the phase transformations, as well as the existence of the intrinsic dissipation. For the governing equations we prove existence, uniqueness and regularity in certain well chosen functions spaces.
\end{abstract}

MSC: 74N30, 74H20, 74H25.

Keywords: shape memory alloys, phase transition, non-isothermal, intrinsic dissipation, existence and uniqueness of solutions.

\section{Introduction}

The thermomechanical model studied in this paper will be published in [3]. It is founded on a free energy which is a convex function with respect to the strain and to the martensitic volume fraction and concave with respect to the temperature. In the present work we prove existence, uniqueness and regularity of solutions in various functions spaces for the circular cylindrical case. Uniqueness in a large class of spaces (abstract derivation structures), as well as existence in the space of continuous functions were established in [5] and [6]. Some of the prerequisites can be found in [5] and $[6]$.

In the circular cylindrical case, the given axial stress $\sigma$, the martensitic volume fraction $\beta$, the variation $\theta$ of the absolute temperature of the

\footnotetext{
${ }^{1}$ École Polytechnique Fédérale de Lausanne, Institut de Mathématiques Bernoulli, 1015 Lausanne, Switzerland. tudor.ratiu@epfl.ch

${ }^{2}$ École Polytechnique Fédérale de Lausanne, Institut de Mathématiques Bernoulli, 1015 Lausanne, Switzerland. aida.timofte@epfl.ch

${ }^{3}$ École Polytechnique Fédérale de Lausanne, Institut de Mathématiques Bernoulli, 1015 Lausanne, Switzerland. vlad.timof te@epfl.ch
} 
sample with respect to $T_{0}$ (temperature of the room), and the axial strain $\varepsilon$ depend only on the time variable $t \in J(J$ interval with $\min J=0)$ and satisfy the following system (see [3] and [5] for details):

$$
(\mathcal{T}):\left\{\begin{array}{c}
\left.\dot{\theta}+\frac{1}{\tau} \theta=\Gamma|\dot{\beta}|+\frac{L}{C} \dot{\beta} \quad \text { (the heat propagation equation }(\mathcal{H})\right) \\
\sigma=E(\varepsilon-g \beta) \quad(\text { the constitutive equation) } \\
0 \leq \beta \leq 1 \\
(\mathcal{E}):\left\{\begin{array}{c}
\text { If } \beta=0, \text { then } \sigma \leq \sigma^{+} \text {and } \\
\dot{\beta}<0 \Rightarrow \sigma \leq \sigma^{-} \\
\text {If } 0<\beta<1, \text { then } \sigma^{-} \leq \sigma \leq \sigma^{+} \text {and } \\
\left\{\begin{array}{l}
\dot{\beta}<0 \Rightarrow \sigma=\sigma^{-} \\
\dot{\beta}>0 \Rightarrow \sigma=\sigma^{+}
\end{array}\right. \\
\text {If } \beta=1, \text { then } \begin{array}{r}
\sigma \geq \sigma^{-} \text {and } \\
\dot{\beta}>0 \Rightarrow \sigma \geq \sigma^{+}
\end{array} \\
\beta(0)=0, \theta(0)=0, \varepsilon(0)=0, \sigma(0)=0,
\end{array}\right.
\end{array}\right.
$$

where $\sigma^{ \pm}:=p\left(T_{0}-T_{\mathrm{a}}+\theta+\beta \Delta T\right) \pm q$. The constants $\tau, \Gamma, L, C, E, g, p, q, T_{0}$, $T_{\mathrm{a}}, \Delta T$ are all positive and $T_{0}>T_{\mathrm{a}}, \Gamma<L / C$. Some comments are necessary in order to understand the mathematical problem raised by $(\mathcal{T})$ :

1. The known data is an arbitrarily given continuous function $\sigma: J \rightarrow \mathbf{R}$ (with $\sigma(0)=0$ ). The system $(\mathcal{T})$ is initially considered for unknown functions $\beta, \theta, \varepsilon: J \rightarrow \mathbf{R}$ having at least lateral derivatives on $J$, since they should satisfy $(\mathcal{H}),(\mathcal{E})$ with respect to these ones. If $\beta$ is strictly increasing on some open subinterval $J_{0} \subset J$, then ${ }^{1}\left\{t \in J_{0} \mid \dot{\beta}_{\mathrm{f}}(t)>0\right\}$ is dense in $J_{0}$, and so $\sigma=\sigma^{+}=p\left(T_{0}-T_{\mathrm{a}}+\theta+\beta \Delta T\right)+q$ on $J_{0}$, by $(\mathcal{E})$. It follows that $\sigma$ should have lateral derivatives on $J_{0}$. This poses a serious compatibility problem for our system if the given $\sigma$ does not have lateral derivatives (e.g. if $\sigma$ is continuous but nowhere differentiable).

2. If $\sigma$ is such that $\dot{\beta}_{\mathrm{b}}\left(t_{0}\right)>0$ and $\beta\left(t_{0}\right)=1$, then $\beta$ cannot be differentiable at $t_{0}$, since $\beta \leq 1$. This may happen even if $\sigma$ is analytic on $J$ and so $\beta$ can be less regular than $\sigma$. This is the reason to insist on lateral differentiability.

3. There exist strictly increasing continuous functions $u: J \rightarrow \mathbf{R}$, such that $\int_{0}^{t} \dot{u}(s) \mathrm{d} s=0 \neq u(t)-u(0)$ for every $t>0$. Since the usual derivative sometimes fails to characterize continuous and almost everywhere differentiable functions, its presence in $(\mathcal{T})$ may not guarantee the uniqueness of solutions.

4. Since for arbitrary given $\sigma$ a pronounced non-differentiability of solutions may occur, it would be natural to study $(\mathcal{T})$ in the space $C(J)$ of all real continuous functions on $J$, with the derivative in the sense of distributions. This is related to serious difficulties: what is the meaning of $|\dot{\beta}|$ in $(\mathcal{H})$ and of $\dot{\beta}(t)$ in $(\mathcal{E})$, if $\dot{\beta}$ is a distribution but not a function?

In order to remove the derivatives of $\beta$ from $(\mathcal{E})$, we introduced in [5] a new notion. A point $t \in J_{0}\left(J_{0}\right.$ an interval) is said to be an increment point for $u \in C\left(J_{0}\right)$, if and only if for every neighborhood $V$ of $t$, we have $t_{1}<t_{2}$ and $u\left(t_{1}\right)<u\left(t_{2}\right)$ for some $t_{1}, t_{2} \in V \cap J_{0}$. Let $M^{+}(u)$ denote the set of all increment points of $u$ and set $M^{-}(u):=M^{+}(-u)$. If $X(J)$ is any of the spaces $B V_{\text {loc }}(J), A C_{\text {loc }}(J), \operatorname{Lip}_{\text {loc }}(J), D_{\mathrm{f}}^{A}(J), D_{\mathrm{b}}^{A}(J), D_{1}^{A}(J), D^{\aleph_{0}}(J)$, $A_{\mathrm{f}}(J), A_{\mathrm{b}}(J), A_{\mathrm{l}}(J)$ endowed with its natural derivative (see the list below for details), then an equivalent form of $(\mathcal{E})$ for $\beta, \theta \in X(J)$ is

\footnotetext{
${ }^{1} \dot{u}_{\mathrm{f}}(t)$ and $\dot{u}_{\mathrm{b}}(t)$ denote the forward and the backward derivatives of $u$ at $t$.
} 


$$
(\mathcal{E})_{X(J)}\left\{\begin{array}{l}
\beta(t)>0 \Rightarrow \sigma(t) \geq \sigma^{-}(t) \\
\beta(t)<1 \Rightarrow \sigma(t) \leq \sigma^{+}(t) \\
t \in M^{+}(\beta) \Rightarrow \sigma(t)=\sigma^{+}(t) \\
t \in M^{-}(\beta) \Rightarrow \sigma(t)=\sigma^{-}(t) .
\end{array}\right.
$$

If $\beta, \theta \in C(J)$ satisfy $(\mathcal{E})_{C(J)}$, then $\beta$ must be locally monotone (see [5], Cor.4.2, p.455). If we write $(\mathcal{H})$ on every interval $J_{0}$ of monotonicity for $\beta$, we can then consider the following equation in distributions on $\stackrel{\circ}{J_{0}}$ :

$$
\dot{\theta}+\frac{1}{\tau} \theta=\left(\Gamma_{0}+\frac{L}{C}\right) \dot{\beta} \quad \text { in } \mathcal{D}^{\prime}\left(\stackrel{o}{J_{0}}\right),
$$

where $\Gamma_{0}:=\left\{\begin{aligned} \Gamma, & \text { if } \beta \text { is increasing on } J_{0} \\ -\Gamma, & \text { else. }\end{aligned}\right.$

The system $(\mathcal{T})$ may be considered for any of the functions spaces and derivatives listed below (see [5] for the definition of an abstract derivation structure $X(J)$ and the corresponding system $\left.(\mathcal{T})_{X(J)}\right)$.

\section{List of functions spaces and associated derivatives}

1) $C(J)$, with the derivative in the sense of distributions in $\mathcal{D}^{\prime}(\stackrel{o}{J})$. We have the natural inclusions $C(J) \subset C(\stackrel{o}{J}) \subset \mathcal{D}^{\prime}(\stackrel{o}{J})$. Let us recall that $u \in C(J)$ is increasing if and only if $u^{\prime} \in \mathcal{D}^{\prime}(\stackrel{o}{J})$ is positive.

2) $B V_{\text {loc }}(J):=\{u \in C(J) \mid u$ has locally bounded variation $\}$, with the usual derivative, that exists almost everywhere.

3) $A C_{\text {loc }}(J):=\{u \in C(J) \mid u$ is locally absolutely continuous $\}$, with the derivative almost everywhere.

4) $\operatorname{Lip}_{\text {loc }}(J):=\{u \in C(J) \mid u$ is locally Lipschitz $\}$, with the derivative almost everywhere.

5) For every fixed at most countable subset $A$ of $J$, consider the spaces: a) $D_{\mathrm{f}}^{A}(J):=\{u \in C(J) \mid u$ is differentiable to the right on $J \backslash A\}$ (respectively $\left.D_{\mathrm{b}}^{A}(J)\right)$, with the forward (respectively backward) derivative on $J \backslash A$.

b) $D_{1}^{A}(J)=D_{\mathrm{f}}^{A}(J) \cap D_{\mathrm{b}}^{A}(J)$, with both forward and backward derivatives. 6) $D^{\aleph_{0}}(J):=\{u \in C(J) \mid$ the set of non-differentiability points of $u$ is at most countable\}, with the usual derivative where this one exists.

7) a) $A_{\mathrm{f}}(J):=\{u \in C(J) \mid u$ is forward-analytic $\}$, with the forward derivative. A function $u \in C(J)$ is said to be forward-analytic at $t \in J \backslash\{\sup J\}$, iff $u$ is analytic on some $[t, s) \subset J(s>t)$. We call $u$ a forward-analytic function, iff $u$ is forward-analytic at every $t \in J \backslash\{\sup J\}$.

b) $A_{\mathrm{b}}(J):=\{u \in C(J) \mid u$ is backward-analytic $\}$, with the backward derivative (definitions are similar to those for $A_{\mathrm{f}}(J)$ ).

c) $A_{\mathrm{l}}(J):=A_{\mathrm{f}}(J) \cap A_{\mathrm{b}}(J)$, with both forward and backward derivatives.

Our problem is the following: for a fixed $X(J)$ in the above list and a given $\sigma \in X(J)$ with $\sigma(0)=0$, we wish to investigate the existence of solutions $\beta, \theta, \varepsilon \in X(J)$ of the system $(\mathcal{T})_{X(J)}$. It is easily seen that the constitutive equation $\sigma=E(\varepsilon-g \beta)$ and the condition $\varepsilon(0)=0$ from $(\mathcal{T})_{X(J)}$ can be ignored. Indeed, if $\beta, \theta \in X(J)$ satisfy all other conditions, we get a solution of $(\mathcal{T})_{X(J)}$ with $\varepsilon=\frac{\sigma}{E}+g \beta \in X(J)$. Therefore, every solution of $(\mathcal{T})_{X(J)}$ is given by a pair $(\beta, \theta)$ of functions from $X(J)$. 


\section{Regularity lemmas}

\subsection{The SMA-optimal function}

Let us consider a non-degenerate interval $J_{0} \subset \mathbf{R}$ with $r:=\min J_{0}$. The SMA linear operators $U_{r}^{0}, V_{r}^{0}: C\left(J_{0}\right) \rightarrow C\left(J_{0}\right)$ are defined by

$$
\begin{aligned}
& U_{r}^{0} v(t)=v(t)+\gamma_{0} \mathrm{e}^{-\alpha_{0} t} \int_{r}^{t} \mathrm{e}^{\alpha_{0} s} v(s) \mathrm{d} s, \\
& V_{r}^{0} u(t)=u(t)-\gamma_{0} \mathrm{e}^{-t / \tau} \int_{r}^{t} \mathrm{e}^{s / \tau} u(s) \mathrm{d} s,
\end{aligned}
$$

where $\alpha_{0}:=\frac{\Delta T}{\tau\left(\Gamma_{0}+L / C+\Delta T\right)}>0, \gamma_{0}:=\frac{1}{\tau}-\alpha_{0}>0, \Gamma_{0} \in\{\Gamma,-\Gamma\}$.

Note that $U_{r}^{0}, V_{r}^{0}$ are invertible and $\left(U_{r}^{0}\right)^{-1}=V_{r}^{0}$ (see [5], Prop.6.1, p.460). For simplicity, we will write $\alpha, \gamma, U_{r}, V_{r}$ instead of $\alpha_{0}, \gamma_{0}, U_{r}^{0}, V_{r}^{0}$, when no confusion can arise or when distinction is not important. These operators are useful in the study of $(\mathcal{T})_{X(J)}$ on intervals of monotonicity for $\beta$. If $J_{0}$ is such an interval, we always take $\Gamma_{0}$ as in (1). If $\Gamma_{0}=\Gamma$, we also use the notations $\alpha_{+}, \gamma_{+}, U_{r}^{+}, V_{r}^{+}$. We shall need the following three results proved in [6] (Lemma 2.1 and Prop.2.1 and 2.2).

Lemma 1 For every $\omega \in C\left(J_{0}\right)$, set

$$
A_{r}^{+}(\omega):=\left\{v \in C\left(J_{0}\right) \mid v(r) \geq 0, v \geq \omega, U_{r} v \text { is increasing }\right\}
$$

and define $w: J_{0} \longrightarrow \mathbf{R}, w(t)=\inf \left\{v(t) \mid v \in A_{r}^{+}(\omega)\right\}$. Then

$$
w \in A_{r}^{+}(\omega), \quad w(r)=\max (\omega(r), 0), \quad M^{+}\left(U_{r} w\right) \subset(w-\omega)^{-1}(\{0\}) .
$$

Note that $w=\min A_{r}^{+}(\omega)$ with respect to the pointwise order relation on $C\left(J_{0}\right)$. Therefore, we call $w$ the SMA-optimal function for $\omega$.

Proposition 1 Let $v \in C\left(J_{0}\right)$, such that $v(r) \geq 0$ and $u=U_{r} v$ is an increasing map. For every fixed $a \in J_{0}$, define the function

$$
\underline{v}_{a}: J_{0} \rightarrow \mathbf{R}, \quad \underline{v}_{a}(t)=\tau \alpha u(a)+[v(a)-\tau \alpha u(a)] \mathrm{e}^{(a-t) / \tau} .
$$

Then we have the following:

1) $v(a)-\tau \alpha u(a) \geq 0$, hence $\underline{v}_{a}$ is a decreasing convex function;

2) For every fixed $t \in J_{0}$, the map $J_{0} \ni a \mapsto \underline{v}_{a}(t) \in \mathbf{R}$ is increasing;

3) $v \leq \underline{v}_{a}$ on $[r, a], v \geq \underline{v}_{a}$ on $[a, \infty) \cap J_{0} a_{a n d}^{2} U_{r}\left(v \wedge \underline{v}_{a}\right)=u \wedge u(a)$;

4) For every subinterval $J_{1} \subset J_{0}$ and every fixed $a \in J_{1}$, the equivalence holds:

$$
u \text { is constant on } J_{1} \Longleftrightarrow v=\underline{v}_{a} \text { on } J_{1} \text {. }
$$

Proposition 2 Let $v \in C\left(J_{0}\right)$ with $v(r) \geq 0$ and $u:=U_{r} v$.

1) If $u$ is increasing, then $J_{0} \ni t \mapsto \mathrm{e}^{\gamma t} v(t) \in \mathbf{R}$ is increasing.

2) If $u$ is increasing on $[r, a]$ and $v$ is increasing on $[a, \infty) \cap J_{0}$ for some $a \in J_{0}$, then $u$ is increasing.

\footnotetext{
${ }^{2}$ We use the notation $u \wedge v$ for the pointwise minimum of two real functions.
} 


\subsection{Regularity of the SMA-optimal function}

In this subsection we shall consider $U_{r}: C\left(J_{0}\right) \rightarrow C\left(J_{0}\right)\left(J_{0}\right.$ interval with $\left.r=\min J_{0}\right)$, a given $\omega \in C\left(J_{0}\right)$, the optimal function $w:=\min A_{r}^{+}(\omega)$, and $u:=U_{r} w$. It is important to note that $w(t)=\omega(t)$ whenever $t \in M^{+}(u)$.

Proposition 3 For every $c \in J_{0}$, we have

$$
A_{r}^{+}\left(\left.\omega\right|_{[r, c]}\right)=\left.A_{r}^{+}(\omega)\right|_{[r, c]}:=\left\{\left.v\right|_{[r, c]} \mid v \in A_{r}^{+}(\omega)\right\} .
$$

In particular, for every $v_{0} \in A_{r}^{+}\left(\left.\omega\right|_{[r, c]}\right)$, we have $v_{0} \geq\left. w\right|_{[r, c]}$.

Proof. The inclusion " $\supset$ " is evident. To prove "C", fix $v_{0} \in A_{r}^{+}\left(\left.\omega\right|_{[r, c]}\right)$ and define the map

$$
v: J_{0} \rightarrow \mathbf{R}, \quad v(t)= \begin{cases}v_{0}(t) & , \quad t \in[r, c] \\ v_{0}(c)+\max _{s \in[c, t]}(\omega(s)-\omega(c)) & , \quad t \geq c .\end{cases}
$$

Since by hypothesis, $v_{0} \in C([r, c]), v_{0}(r) \geq 0, v_{0} \geq\left.\omega\right|_{[r, c]}$ and $U_{r} v_{0}$ is increasing, we see that $v \in C\left(J_{0}\right), v(r) \geq 0, v \geq \omega$. As $U_{r} v$ is increasing on $[r, c]$ and $v$ is increasing on $[c, \infty) \cap J_{0}$, we deduce that $v \in A_{r}^{+}(\omega)$, by Proposition 2(2). We thus get $v_{0}=\left.\left.v\right|_{[r, c]} \in A_{r}^{+}(\omega)\right|_{[r, c]}$ and $v \geq w$.

Lemma 2 If $\omega$ is differentiable to the right at $a \in J_{0}$, then so is $w$ and

$$
\dot{w}_{\mathrm{f}}(a)= \begin{cases}\dot{w}_{a}(a), & \text { if } u \equiv u(a) \text { on some }[a, c] \subset J_{0}, c>a, \\ \dot{\omega}_{\mathrm{f}}(a), & \text { if } u(t)>u(a) \forall t>a .\end{cases}
$$

Proof. Fix $a \in J_{0}$, such that $\dot{\omega}_{\mathrm{f}}(a)$ exists. We need to consider two cases: 1 ) If $u \equiv u(a)$ on some $[a, c] \subset J_{0}$ with $c>a$, then Proposition 1 (4) shows that $w=\underline{w}_{a}$ on $[a, c]$, and so $\exists \dot{w}_{\mathrm{f}}(a)=\underline{\dot{w}}_{a}(a)=\lambda u(a)-\tau^{-1} w(a)$.

2) If $u(t)>u(a) \forall t>a$, then $a \in M^{+}\left(\left.u\right|_{I}\right)$, where $I:=[a, \infty) \cap J_{0}$. Since $M^{+}\left(\left.u\right|_{I}\right)$ is a perfect set in $I$ (see [5], Prop.4.1(3), p.453), there exists a sequence $\left(t_{n}\right)_{n \in \mathbf{N}} \subset M^{+}\left(\left.u\right|_{I}\right) \backslash\{a\} \subset M^{+}(u)$, with $t_{n} \rightarrow a$. By Lemma 1 and Proposition 1(3), we have $\omega\left(t_{n}\right)=w\left(t_{n}\right) \geq \underline{w}_{a}\left(t_{n}\right) \forall n \in \mathbf{N}$. Therefore, $\omega(a)=w(a)=\underline{w}_{a}(a)$ leads to

$$
\dot{\omega}_{\mathrm{f}}(a)=\lim _{n \rightarrow \infty} \frac{\omega\left(t_{n}\right)-\omega(a)}{t_{n}-a} \geq \lim _{n \rightarrow \infty} \frac{\underline{w}_{a}\left(t_{n}\right)-\underline{w}_{a}(a)}{t_{n}-a}=\underline{\dot{w}}_{a}(a) .
$$

Now fix $\xi>\dot{\omega}_{\mathrm{f}}(a) \geq \underline{\dot{w}}_{a}(a)$, choose $c \in J_{0}$ such that $c>a$ and

$$
\omega(t)<\omega(a)+\xi(t-a), \quad \underline{\dot{w}}_{a}(t)<\xi \quad \forall t \in(a, c],
$$

and define the map

$$
v_{0}:[r, c] \rightarrow \mathbf{R}, \quad v_{0}(t)= \begin{cases}w(t) & , \quad t \in[r, a] \\ \omega(a)+\xi(t-a) & , \quad t \in[a, c]\end{cases}
$$

Obviously, $v_{0} \in C([r, c]), v_{0}(r)=w(r) \geq 0$ and $v_{0} \geq\left.\omega\right|_{[r, c]}$. We claim that $U_{r} v_{0}$ is increasing. To prove this, define $\varphi=v_{0}-w \wedge \underline{w}_{a} \in C([r, c])$, that is, $\varphi \equiv 0$ on $[r, a]$ and $\varphi=v_{0}-\underline{w}_{a}$ on $[a, c]$, by Proposition $1(3)$. But $\dot{\varphi} \equiv 0$ on $[r, a)$ and $\dot{\varphi}=\xi-\underline{\dot{w}}_{a}>0$ on $(a, c]$, and so $\varphi$ is increasing. As $\varphi(r)=0$, Proposition 2(2) shows that $U_{r} \varphi$ is increasing. Since by Proposition 1(3)

$$
U_{r} v_{0}=U_{r} \varphi+U_{r}\left(w \wedge \underline{w}_{a}\right)=U_{r} \varphi+u \wedge u(a),
$$


the map $U_{r} v_{0}$ is increasing. It follows that $v_{0} \in A_{r}^{+}\left(\left.\omega\right|_{[r, c]}\right)$, which gives $\omega \leq w \leq v_{0}$ on $[r, c]$, by Proposition 3. This and $\omega(a)=w(a)=v_{0}(a)$ yield

$$
\dot{\omega}_{\mathrm{f}}(a) \leq \liminf _{t \searrow a} \frac{w(t)-w(a)}{t-a} \leq \limsup _{t \searrow a} \frac{w(t)-w(a)}{t-a} \leq \dot{v}_{\mathrm{f}}(a)=\xi .
$$

Since $\xi>\dot{\omega}_{\mathrm{f}}(a)$ was arbitrary, we conclude that $\exists \dot{w}_{\mathrm{f}}(a)=\dot{\omega}_{\mathrm{f}}(a)$.

Lemma 3 If $\omega$ is differentiable to the left at $a \in J_{0}$, then so is $w$ and

$$
\dot{w}_{\mathrm{b}}(a)= \begin{cases}\dot{w}_{a}(a) \quad, \quad \text { if } u \equiv u(a) \text { on some }[c, a] \subset J_{0}, c<a, \\ \dot{\omega}_{\mathrm{b}}(a) \quad, \quad \text { if } u(t)<u(a) \forall t \in[r, a) .\end{cases}
$$

Proof. Fix $a \in J_{0}$, such that $\dot{\omega}_{\mathrm{b}}(a)$ exists. We need to consider two cases: 1) If $u \equiv u(a)$ on some $[c, a] \subset J_{0}$ with $c<a$, then Proposition 1 (4) shows that $w=\underline{w}_{a}$ on $[c, a]$, and so $\exists \dot{w}_{\mathrm{b}}(a)=\underline{\dot{w}}_{a}(a)=\lambda u(a)-\tau^{-1} w(a)$.

2) If $u(t)<u(a) \forall t \in[r, a)$, then $a \in M^{+}\left(\left.u\right|_{[r, a]}\right) \subset M^{+}(u)$, and so $\omega(a)=w(a)$. Now fix $\xi<\dot{\omega}_{\mathrm{b}}(a)$ and define the maps

$$
\varphi, \chi:[r, a) \rightarrow \mathbf{R}, \quad \varphi(t)=\frac{w(a)-w(t)}{a-t}, \quad \chi(t)=\frac{\omega(a)-\omega(t)}{a-t} .
$$

We have $\varphi \leq \chi$. Since $M^{+}\left(\left.u\right|_{[r, a]}\right)$ is a perfect set in $[r, a]$ (see [5], Prop. 4.1(3), p.453), choose $c \in M^{+}(u) \cap[r, a)$, with $\chi(t)>\xi \forall t \in[c, a)$. We claim that $\varphi>\xi$ on $[c, a)$. To prove this, fix $t_{0} \in[c, a)$. We need to consider two subcases:

i) If $t_{0} \in M^{+}(u)$, then $w\left(t_{0}\right)=\omega\left(t_{0}\right)$ yields $\varphi\left(t_{0}\right)=\chi\left(t_{0}\right)>\xi$.

ii) If $t_{0} \notin M^{+}(u)$, set $d:=\sup \left(M^{+}(u) \cap\left[c, t_{0}\right]\right) \neq \emptyset$. We have $d \in M^{+}(u)$, $w(d)=\omega(d), c \leq d<t_{0}$ and $\left.u\right|_{\left[d, t_{0}\right]} \equiv u(d)$, since $M^{+}(u) \cap\left(d, t_{0}\right)=\emptyset$. We thus get $w=\underline{w}_{d}$ on $\left[d, t_{0}\right]$, by Proposition 1(4). Hence, $\varphi$ is differentiable on $\left(d, t_{0}\right)$. As Proposition $1(2)$ gives $w(a)=\underline{w}_{a}(a) \geq \underline{w}_{d}(a)$, we obtain

$$
\dot{\varphi}(t)=\frac{w(a)-w(t)-\dot{w}(t)(a-t)}{(a-t)^{2}} \geq \frac{\underline{w}_{d}(a)-\underline{w}_{d}(t)-\underline{\dot{w}}_{d}(t)(a-t)}{(a-t)^{2}} \geq 0
$$

on $\left(d, t_{0}\right)$, because $\underline{w}_{d}$ is a convex map, according to Proposition $1(1)$. It follows that $\varphi\left(t_{0}\right) \geq \varphi(d)=\chi(d)>\xi$.

From (i) and (ii), we conclude that $\chi \geq \varphi>\xi$ on $[c, a)$. Therefore,

$$
\xi \leq \liminf _{t \nearrow a} \varphi(t) \leq \limsup _{t \nearrow a} \varphi(t) \leq \limsup _{t / a} \chi(t)=\dot{\omega}_{\mathrm{b}}(a)
$$

Since $\xi<\dot{\omega}_{\mathrm{b}}(a)$ was arbitrary, we conclude that $\exists \dot{w}_{\mathrm{b}}(a)=\dot{\omega}_{\mathrm{b}}(a)$.

Lemma 4 If $\omega \in D^{\aleph_{0}}\left(J_{0}\right)$, then $w \in D^{\aleph_{0}}\left(J_{0}\right)$.

Proof. Let $N(\omega)$ and $N(w)$ denote the sets of points where $\omega$ and $w$ are not differentiable. It suffices to show that $N(w) \backslash N(\omega)$ is at most countable. By Lemmas 2 and 3, we deduce that each $a \in N(w) \backslash N(\omega)$ must belong to the boundary of some maximal non-degenerate subinterval of $I_{a}$, on which $u$ is constant ( $I_{a}$ is maximal with this property). Since by Lindelöf's theorem, the set of all such intervals is at most countable, the conclusion follows immediately.

Lemma 5 If $\omega$ is forward-analytic at $a \in J_{0}$, then so is $w$. 
Proof. Fix $a \in J_{0}$, such that $\omega$ is forward-analytic at $a$. We need to consider two cases:

1) If $u \equiv u(a)$ on some $[a, c] \subset J_{0}$ with $c>a$, then Proposition $1(4)$ shows that $w=\underline{w}_{a}$ on $[a, c]$, and so $w$ is forward-analytic at $a$.

2) If $u(t)>u(a) \forall t>a$, then $a \in M^{+}\left(\left.u\right|_{[a, \infty) \cap J_{0}}\right) \subset M^{+}(u)$, and so $\omega(a)=w(a)$. As $\omega$ is forward-analytic at $a$, the map $\omega-\underline{w}_{a}$ must be analytic and monotone on some $[a, c] \subset J_{0}, c>a$. We next show that $(\omega-$ $\left.\underline{w}_{a}\right)\left.\right|_{[a, c]}$ is increasing. Suppose to the contrary that this map is decreasing, and hence $\omega \leq \underline{w}_{a}$ on $[a, c]$. Define $v_{0}:=\left.\left(w \wedge \underline{w}_{a}\right)\right|_{[r, c]} \in C([r, c])$. By Proposition 1(3), it follows that $v_{0} \in A_{r}^{+}\left(\left.\omega\right|_{[r, c]}\right)$, and so $v_{0} \geq\left. w\right|_{[r, c]}$, by Proposition 3. On $[a, c]$, we have $\underline{w}_{a} \leq w \leq v_{0}=\underline{w}_{a}$, hence $w=\underline{w}_{a}$, and finally $u \equiv u(a)$ (which is a contradiction), by Proposition $1(3,4)$. We conclude that $\left.\left(\omega-\underline{w}_{a}\right)\right|_{[a, c]}$ is increasing. Now define

$$
v_{1}:[r, c] \rightarrow \mathbf{R}, \quad v_{1}(t)= \begin{cases}w(t) & , \quad t \in[r, a] \\ \omega(t) \quad, \quad t \in[a, c] .\end{cases}
$$

Clearly, $v_{1} \in C([r, c]), v_{1}(r) \geq 0, v_{1} \geq\left.\omega\right|_{[r, c]}$. For $v_{0}$ defined as above, $\left(v_{1}-v_{0}\right)(r)=0$, the map $v_{1}-v_{0}$ is increasing, and so $U_{r}\left(v_{1}-v_{0}\right)$ is increasing, by Proposition 2. As $U_{r} v_{0}$ is increasing, we deduce that so is $U_{r} v_{1}$. It follows that $v_{1} \in A_{r}^{+}\left(\left.\omega\right|_{[r, c]}\right)$, and consequently $v_{1} \geq\left. w\right|_{[r, c]}$, by Proposition 3. On $[a, c]$, we have $\omega \leq w \leq v_{1}=\omega$, and hence $w=\omega$. We conclude that $w$ is forward-analytic at $a$.

Lemma 6 If $\omega$ is backward-analytic at $a \in J_{0}$, then so is $w$.

Proof. Fix $a \in J_{0}$, such that $\omega$ is backward-analytic at $a$. We need to consider two cases:

1) If $u \equiv u(a)$ on some $[c, a] \subset J_{0}$ with $c<a$, then Proposition 1 (4) shows that $w=\underline{w}_{a}$ on $[c, a]$, and so $w$ is backward-analytic at $a$.

2) If $u(t)<u(a)$, for every $t \in[r, a)$, then $a \in M^{+}\left(\left.u\right|_{[r, a]}\right) \subset M^{+}(u)$, and so $\omega(a)=w(a)$. On $[r, a]$, we have $\omega \leq w \leq \underline{w}_{a}$, by Proposition 1(3). Define $\varphi:[r, a] \rightarrow \mathbf{R}, \varphi(t)=\mathrm{e}^{t / \tau}\left(\underline{w}_{a}(t)-\omega(t)\right)$. Since $\varphi \geq 0=\varphi(a)$ and $\varphi$ is backward-analytic at $a$, the map $\varphi$ must be analytic and decreasing on some $[c, a] \subset J_{0}, c<a$. As $M^{+}\left(\left.u\right|_{[r, a]}\right)$ is a perfect set in $[r, a]$, choose $d \in M^{+}(u) \cap[c, a) \neq \emptyset$. Hence, $w(d)=\omega(d)$. Now define

$$
v_{0}:[r, a] \rightarrow \mathbf{R}, \quad v_{0}(t)= \begin{cases}w(t) & , \quad t \in[r, d] \\ \omega(t) & , \quad t \in[d, a] .\end{cases}
$$

Clearly, $v_{0} \in C([r, a]), v_{0}(r) \geq 0, v_{0} \geq\left.\omega\right|_{[r, a]}$. We claim that $u_{0}:=U_{r} v_{0}$ is increasing. On $[r, d], u_{0}=U_{r} w=u$ is increasing. On $(d, a)$, we have

$$
\varphi \text { is decreasing } \Longleftrightarrow \dot{\varphi} \leq 0 \Longleftrightarrow \dot{\omega}+\frac{1}{\tau} \omega \geq \underline{\dot{w}}_{a}+\frac{1}{\tau} \underline{w}_{a} \equiv \lambda u(a),
$$

the last equality being easily checked. But $u_{0}=U_{r} v_{0}$ also gives on $(d, a)$ (see [5], Rem.6.2, p.461)

$$
\dot{u}_{0}+\lambda u_{0}=\dot{v}_{0}+\frac{1}{\tau} v_{0}=\dot{\omega}+\frac{1}{\tau} \omega \geq \lambda u(a)>\lambda u,
$$

which yields $\dot{u}_{0}>\lambda\left(u-u_{0}\right)=\lambda U_{r}\left(w-v_{0}\right) \geq 0$ on $(d, a)$, since $\left.w\right|_{[r, a]} \geq v_{0}$ and $U_{r}$ is a positive operator on $C([r, a])$. Since $u_{0}$ is increasing on both $[r, d]$ and $[d, a]$, it is so on $[r, a]$. It follows that $v_{0} \in A_{r}^{+}\left(\left.\omega\right|_{[r, a]}\right)$, and hence $v_{0} \geq\left. w\right|_{[r, a]}$, by Proposition 3. On $[d, a]$, we have $\omega \leq w \leq v_{0}=\omega$, and consequently $w=\omega$. We conclude that $w$ is backward-analytic at $a$. 
Proposition 4 For all $a, b \in J_{0}, a \leq b$, we have

$$
-\gamma w(a)(b-a) \leq w(b)-w(a) \leq \max _{t \in[a, b]}(\omega(t)-\omega(a)) .
$$

Proof. Fix $a, b \in J_{0}, a \leq b$. By Proposition 2(1), we have

$$
w(a)-w(b) \leq w(a)\left(1-\mathrm{e}^{\gamma(a-b)}\right) \leq \gamma w(a)(b-a) .
$$

To prove the other inequality, define

$$
v: J_{0} \rightarrow \mathbf{R}, \quad v(t)= \begin{cases}w(t) & , \quad t \in[r, a] \\ w(a)+\max _{s \in[a, t]}(\omega(s)-\omega(a)) & , \quad t \geq a .\end{cases}
$$

As in the proof of Proposition 3, it follows that $v \in A_{r}^{+}(\omega)$, and so $v \geq w$. Now $v(b) \geq w(b)$ yields the claimed inequality.

The proofs of the following two lemmas are based on Proposition 4 .

Lemma 7 If $\omega \in \operatorname{Lip}_{\text {loc }}\left(J_{0}\right)$, then $w \in \operatorname{Lip}_{\text {loc }}\left(J_{0}\right)$.

Lemma 8 If $\omega \in A C_{\mathrm{loc}}\left(J_{0}\right)$, then $w \in A C_{\mathrm{loc}}\left(J_{0}\right)$.

Proof. Fix $c \in J_{0}$ with $c>r$ and set $I:=[r, c]$. Let $X$ be one of the vector spaces $\operatorname{Lip}(I), A C(I)$. It is required to prove that if $\omega \in X$, then $w \in X$. By (2), we have $w \leq M:=w(r)+\max _{t \in[r, c]}(\omega(t)-\omega(r))$ on $I$, and consequently

$$
|w(b)-w(a)| \leq \gamma M(b-a)+\max _{t \in[a, b]}|\omega(t)-\omega(a)| \quad \forall a, b \in I, a \leq b .
$$

We need to consider two cases:

1) If $X=\operatorname{Lip}(I)$ and $L$ is a Lipschitz constant for $\omega$, then (3) shows that $\gamma L+M$ is a Lipschitz constant for $w$, and so $w \in \operatorname{Lip}(I)=X$.

2) If $X=A C(I)$, fix $\epsilon>0$ and choose $\delta>0$, such that for every finite family $\left\{\left(a_{\lambda}, b_{\lambda}\right)\right\}_{\lambda \in \Lambda}$ of pairwise disjoint subintervals of $I$, we have

$$
\sum_{\lambda \in \Lambda}\left(b_{\lambda}-a_{\lambda}\right)<\delta \Rightarrow \sum_{\lambda \in \Lambda}\left|\omega\left(b_{\lambda}\right)-\omega\left(a_{\lambda}\right)\right|<\frac{\epsilon}{2} .
$$

Obviously, we can choose $\delta$ with $2 \gamma M \delta<\epsilon$. This leads by (3) and (4) to

$$
\sum_{\lambda \in \Lambda}\left(b_{\lambda}-a_{\lambda}\right)<\delta \Rightarrow \sum_{\lambda \in \Lambda}\left|w\left(b_{\lambda}\right)-w\left(a_{\lambda}\right)\right|<\gamma M \delta+\frac{\epsilon}{2}<\epsilon .
$$

We conclude that $w \in A C(I)=X$.

Lemma 9 We have $w \in B V_{\mathrm{loc}}\left(J_{0}\right)$.

Proof. As $U_{r} w=u \in B V_{\text {loc }}\left(J_{0}\right)$, we have $w=V_{r} u \in B V_{\mathrm{loc}}\left(J_{0}\right)$, since $\left(U_{r}\right)^{-1}=V_{r}$ and $B V_{\text {loc }}\left(J_{0}\right)$ is clearly invariant for $V_{r}$.

\section{Existence, uniqueness and regularity}

\subsection{Equivalence of existence and regularity}

Let us first recall the following result (see [5], Cor.5.4, p.460).

Corollary 1 If $X(J)$ is an abstract derivation structure and $\beta, \theta \in C(J)$, then the following statements are equivalent:

1) $(\beta, \theta)$ is a solution of $(\mathcal{T})_{X(J)}$.

2) $(\beta, \theta)$ is a solution of $(\mathcal{T})_{C(J)}$ and $\beta, \theta \in X(J)$. 
Now let $\sigma \in X(J)$ be fixed, such that $\sigma(0)=0$. Since every solution of $(\mathcal{T})_{X(J)}$ also satisfies $(\mathcal{T})_{C(J)}$, we deduce that $(\mathcal{T})_{X(J)}$ is compatible if and only if the unique solution $(\beta, \theta)$ of $(\mathcal{T})_{C(J)}$ (see [6], Th.3.1, p.543) satisfies $\beta, \theta \in X(J)$. Hence, for our problem, regularity of solutions $(\beta, \theta \in X(J)$ whenever $\sigma \in X(J))$ is equivalent to their existence.

\subsection{Existence and uniqueness}

Let $X(J)$ be any of the spaces

$$
\begin{aligned}
& B V_{\mathrm{loc}}(J), A C_{\mathrm{loc}}(J), \operatorname{Lip}_{\mathrm{loc}}(J), D_{\mathrm{f}}^{A}(J), D_{\mathrm{b}}^{A}(J), D_{\mathrm{l}}^{A}(J), \\
& D^{\aleph_{0}}(J), A_{\mathrm{f}}(J), A_{\mathrm{b}}(J), A_{\mathrm{l}}(J),
\end{aligned}
$$

endowed with its natural derivative.

Proposition 5 Let $u: J \rightarrow \mathbf{R}$.

1) If $u \in X(J)$, then $u \in X\left(J_{0}\right)$ for every subinterval $J_{0} \subset J$.

2) If $u \in X(J \cap(-\infty, r])$ and $u \in X(J \cap[r, \infty))$ for some $r \in J$, then $u \in X(J)$.

3) $u \in X(J)$ if and only if for every $t_{0} \in J$, we have $u \in X(W)$ for some $W=J \cap[a, b]$, with $a, b \in \mathbf{R}, a<t_{0}<b$.

Proof. The proof is straightforward for all spaces from (5).

Theorem 1 The system $(\mathcal{T})_{X(J)}$ has a unique solution.

Proof. Fix $\sigma \in X(J) \subset C(J)$ and consider the unique solution $(\beta, \theta)$ of $(\mathcal{T})_{C(J)}$. According to Corollary 1 , it suffices to show that $\beta, \theta \in X(J)$. Let $t_{0} \in J$ be fixed. We shall next prove that $\beta \in X(W)$ for some $W$ as in Proposition 5(3). We need to analyze two cases.

1) If $t_{0} \notin M^{+}(\beta) \cup M^{-}(\beta)$, then $\beta$ is constant on some neighborhood $W=J \cap\left[t_{0}-\delta, t_{0}+\delta\right](\delta>0)$ of $t_{0}$ in $J$, and so $\beta \in X(W)$.

2) If $t_{0} \in M^{+}(\beta)$, then $\sigma\left(t_{0}\right)=\sigma^{+}\left(t_{0}\right)$ yields $t_{0}>0$, since $\sigma(0)<\sigma^{+}(0)$. As $\sigma\left(t_{0}\right) \neq \sigma^{-}\left(t_{0}\right)$, we have $t_{0} \notin M^{-}(\beta)$, and so $\beta$ is increasing on some $W=J \cap[r, s]\left(0 \leq r<t_{0}<s\right)$. We now consider two subcases.

i) If $\beta\left(t_{0}\right)<1$, we can assume that $\beta<1$ on $W$, since $\beta$ is continuous. Thus, on the interval $W$, we have $\sigma \leq \sigma^{+}$and $\beta$ is increasing. It follows that $\sigma^{+}-\sigma_{r}^{+}=\min A_{r}^{+}\left(\sigma-\sigma_{r}^{+}\right)$on $W$ (see [5], Th.7.1, p.463), where $\sigma_{r}^{+}: J \rightarrow \mathbf{R}, \sigma_{r}^{+}(t):=p\left(T_{0}-T_{\mathrm{a}}+\theta(r) \mathrm{e}^{(r-t) / \tau}+\beta(r) \Delta T\right)+q$. But $\sigma_{r}^{+} \in X(W)$ (every abstract derivation space contains the restrictions of analytic functions defined on $\mathbf{R}$; see [5], Def.5.1, p.456 for details) yields $\sigma-\sigma_{r}^{+} \in X(W)$, which leads by Lemmas 2-9 to $\sigma^{+}-\sigma_{r}^{+} \in X(W)$. That $\beta \in X(W)$ follows from the equalities

$$
\sigma^{+}-\sigma_{r}^{+}=\frac{p \Delta T}{\tau \alpha_{+}} V_{r}^{+}(\beta-\beta(r)), \quad \beta-\beta(r)=\frac{\tau \alpha_{+}}{p \Delta T} U_{r}^{+}\left(\sigma^{+}-\sigma_{r}^{+}\right)
$$

(see [5], Cor.5.1 and Not.5.1, p.459), because every abstract derivation space is invariant for the SMA linear operators (see [5], Rem.6.1, p.461). ii) If $\beta\left(t_{0}\right)=1$, then $\beta \equiv 1$ on $W \cap\left[t_{0}, s\right]$, and so $\beta \in X\left(W \cap\left[t_{0}, s\right]\right)$. As $t_{0} \in M^{+}(\beta)$, we must have $\beta<1$ on $\left[r, t_{0}\right) \subset W$, and consequently, $\sigma \leq \sigma^{+}$on $\left[r, t_{0}\right]$. As in the previous subcase, it follows that $\beta \in X\left(\left[r, t_{0}\right]\right)$. This and $\beta \in X\left(W \cap\left[t_{0}, s\right]\right)$ lead by Proposition $5(2)$ to $\beta \in X(W)$.

3) If $t_{0} \in M^{-}(\beta)$, an analysis similar to that in the previous case shows that $\beta \in X(W)$ for some $W=J \cap[r, s]\left(0 \leq r<t_{0}<s\right)$.

We conclude that $\beta \in X(W)$ for some neighborhood $W=[r, b]$ of $t_{0}$ in $J$, 
with $\beta$ monotone on $W$. Thus, solving (1) on $J_{0}=W$ gives $\theta \in X(W)$. Since $t_{0}$ was arbitrary, applying Proposition 5(3) shows that $\beta, \theta \in X(J)$.

】

The above existence and uniqueness theorem shows that all $X(J)$ from (5) are natural spaces for the system $(\mathcal{T})_{X(J)}$. It is worth pointing out that in all these cases the resulting problems are distinct and that the system is verified (by its solutions) with respect to a prescribed derivative.

Acknowledgements. T. R. and A. T. thank the Swiss National Science Foundation for partial support.

\section{References}

[1] X. Balandraud, E. Ernst and E. Soós, Relaxation and creep phenomena in shape memory alloys, Part I: Hysteresis loop and pseudoelastical behavior, Z. Angew. Math. Phys. (ZAMP) 51 (2000), 171-203.

[2] X. Balandraud, E. Ernst and E. Soós, Relaxation and creep phenomena in shape memory alloys, Part II: Stress relaxation and strain creep during phase transformation, Z. Angew. Math. Phys. (ZAMP) $\mathbf{5 1}$ (2000), 419-448.

[3] X. Balandraud, E. Ernst and E. Soós, Monotone strain-stress models for Shape Memory Alloys hysteresis loop and pseudoelastic behavior, Z. Angew. Math. Phys. (ZAMP) 55 (2004) (to appear).

[4] A. Timofte and V. Timofte, Qualitative results concerning the behavior of shape memory alloys, Rev. Roumaine Math. Pures Appl. 47 (2002), no.1, 121-133.

[5] A. Timofte and V. Timofte, Uniqueness theorem for a thermomechanical model of shape memory alloys, Math. Mech. Solids 6 (2001), no.4, 447-466.

[6] A. Timofte and V. Timofte, Existence theorem for a thermomechanical model of shape memory alloys, Math. Mech. Solids 6 (2001), no.5, 541545 . 